\title{
INACTIVATION OF CANDIDA ALBICANS BY CORONA DISCHARGE: THE INCREASE OF INHIBITION ZONES AREA AFTER FAR SUBSEQUENT EXPOSITION
}

\author{
Vladyslava FAntova $^{a, *}$, Karol BujaČEK $^{a}$, VÍtĚZSLAV KŘÍHA $^{a}$, \\ JAROSLAV JULÁK ${ }^{b}$ \\ ${ }^{a}$ Czech Technical University in Prague, Fac. of Electrical Engineering, Dep. of Physics, Prague, Czech Republic \\ ${ }^{b}$ Charles University in Prague, First Faculty of Medicine, Inst. of Immunology and Microbiology, Czech Republic \\ * corresponding author: demchvla@fel.cvut.cz
}

\begin{abstract}
The cold atmospheric pressure plasma generated by the negative corona discharge has inhibition effect on the microorganism growth. This effect is well-known and it can be demonstrated on the surface of cultivation agar plates by the formation of inhibition zones. We exposed the cultures of Candida albicans to the negative corona discharge plasma in a special arrangement in this study: The equal doses of plasma were applied subsequently twice or four times on the same culture on one Petri dish, while the distance between exposed points was variable.

Only small differences were observed in decontaminated zone areas for twice exposed agar at the shortest distance between exposed points $(1.5 \mathrm{~cm})$. In case of the four times exposed agars, we observed significant differences in inhibition zone areas, dependent not only on the exposition site distances, but also on the exposition order. The largest inhibition zone size was observed for the first exposition decreasing to the fourth one. To check relevancy of these dependencies, we presume to conduct further set of experiments with lower yeast concentration.

In conclusion, significant difference in partial inhibition zone sizes appeared only when four expositions on one Petri dish were carried out, whereas no significant difference was observed for two subsequent expositions. The explanation of this effect may be the subject of subsequent remote exposition(s), when minute amounts of scattered active particles act on the previously exposed areas; the influence of diffused ozone may also take place.
\end{abstract}

KEYwORDS: cold atmospheric plasma decontamination, Candida albicans, direct and indirect treatment.

\section{INTRODUCTION}

A novel discipline plasma medicine including topics of bio-decontamination and sterilization rapidly develops nowadays. There are many papers concerning interaction between cold atmospheric pressure plasma (CAP) and biological materials (bacteria, yeasts, spores, human and/or animal tissue). It is widely accepted the UV radiation, charged particles, reactive nitrogen and oxygen species are the main plasma-generated factors that affect biological materials [4, 3]. Nevertheless, there is still a lot of ambiguity about exact inactivation mechanisms, dominant factors and their mutual effects, where the influence of the UV radiation is the most unexplored factor. On the one hand, it is well known that UV radiation has germicidal effect and may cause double strain DNA break. On the other hand, some papers declare that there is small or marginal impact of the UV radiation generated in CAP. Some papers even declare that UV-free plasmas have germicidal properties. However, the synergy of UV radiation and reactive species give better decontamination results $9,5,6$.

There are also efforts to separate and study particular factors, or at least to separate direct and indirect
CAP treatment. During the direct treatment, the contaminated object is in direct contact with plasma active (light-emitting) zone, while during the indirect treatment the object is in contact only with longliving products of plasma chemical reactions 10, 2. Current experiments assume mutual or separated influence of individual factors. We focused on separation of near and far decontamination effects using several expositions with different spatial and time arrangement in our experiments.

Common antimicrobial susceptibility tests (antimicrobial gradient method, disc diffusion test) are often realized for several concentrations of antibiotics on one Petri dish [1. Using only one Petri dish is beneficial, as it guarantees the same conditions (inoculum concentration, cultivation medium properties, incubation period, etc.), as well as cost and labor saving. It is important to know whether similar susceptibility test arrangements can be realized with CAP treatment instead of antibiotic application.

The effect of particular factors and components of CAP decrease with distance. According to this, we can distinguish near and far treatment. The near treatment means direct interaction between CAP and 
treated surface, whereas the far treatment means interaction between long living species or highly mobile ones outside the active plasma region.

The near and far treatment effects cannot be distinguished when performing only one experiment on a single Petri dish, while performing subsequent expositions at different places on a single Petri dish, when the distance between exposed areas are greater than inhibition zone of single exposition, enables us to study the near and far treatment superposition.

Experiments presented in this paper are aimed to investigate mutual influence of several expositions on a single Petri dish. The impact of chosen treatment procedure (near treatment followed by the far one and vice versa) also has to be considered. It seems that there is distinguishable effect of chosen order, so near and far treatments are not commutable in decontamination processes.

\section{SAMPLES}

Candida albicans yeast was used as a model microorganism. C. albicans occurs as a commensal in human digestive tract and other parts of human body. In most cases it is nonpathogenic. Potential overgrowth can be observed if the immunity system of host is weakened, for example in case of young or old, HIV infected or hospitalized people. This microorganism can be grown in laboratory conditions without any considerable difficulties. Sabouraud agar, developed for fungi and yeast cultivation, was used as a growth medium. C. albicans suspension in sterile water was spill onto agar surface and more or less uniform layer on surface was created. Resulting concentration of C. albicans was estimated as in order of $10^{8} \mathrm{CFU} \mathrm{cm}^{-2}$ (colony forming units per square $\mathrm{cm}$ ) in this layer. The inoculum concentration and colony confluence are not crucial for the inhibition zone areas, as described in $[7$ or 8 . From the practical point of view, the higher inoculum concentrations are preferable, as they provide sharp zone edges, whereas the lower ones yield not sharply delineated and thus hardly measurable zones.

\section{EXPERIMENTS}

\subsection{Apparatus}

Low temperature plasma was generated by the DC negative corona discharge in the simple point-to-plane electrode system (Figs. 11,2). The high voltage power supply UTES HT 2103 and a ballast resistor (discharge stabilization, $10 \mathrm{M} \Omega$ ) were used. The plane electrode was realized by the Sabouraud agar in Petri dish $(\varnothing 9 \mathrm{~cm})$, which is an ion conducting medium. It was connected to electric circuit by immersed metallic strip. The point electrode was realized by a sharpened brass rod. Polarity of the point electrode was negative, i.e. negative corona discharge was ignited in the electrode gap.

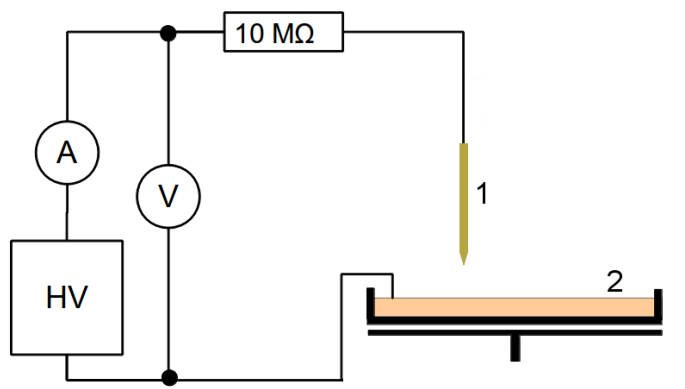

FiguRE 1. Experiment scheme: ammeter A, voltmeter $\mathrm{V}$, power supply $\mathrm{HV}$, ballast resistor $10 \mathrm{M} \Omega$, a point metallic electrode 1 and agar surface as an electrically conductive plane electrode 2 .

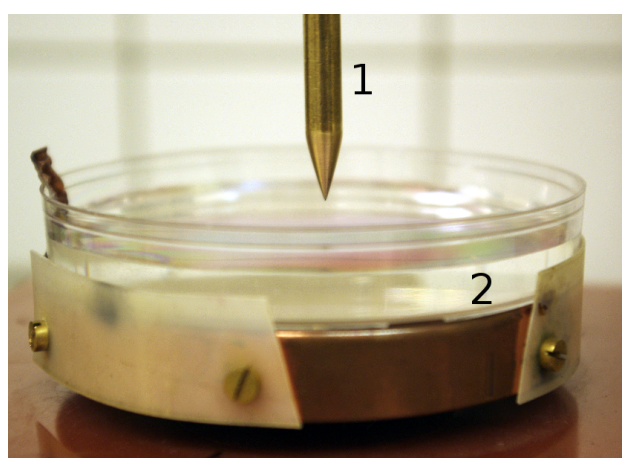

Figure 2. Point electrode 1, Petri dish with ion conductive medium 2 .

Petri dish was placed on rotating stand, enabling us to perform subsequent exposition with fixed radial distance on the same Petri dish.

\subsection{EXPOSITION}

Presuming that multiple expositions are affecting each other, two types of experiments with variable distance between expositions were arranged:

(1.) Two subsequent expositions on one Petri dish (Fig. 3a). Five different distances between opposite exposition locations were used.

(2.) Four subsequent expositions on one Petri dish (Fig. 3p).

The following experimental conditions were used: the gap between electrodes was $7 \mathrm{~mm}$, the exposition time was $5 \mathrm{~min}$, the voltage was set to $10 \mathrm{kV}$ (measured on the power supply) and the resulting electric current was approximately $50 \mu \mathrm{A}$. The current varied due to increasing of gap size, caused by an ion wind causing the agar surface deformation. Exposed dishes were cultivated overnight at $37^{\circ} \mathrm{C}$. Area of inhibition zones was measured manually using the millimeter paper grid.

\section{Results and Discussion}

The inhibition zones were compared for both experiments and are presented in the following paragraphs. Experiments, that are presented here were repeated 


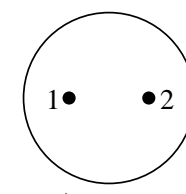

a)

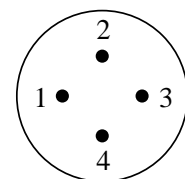

b)
FiguRE 3. Exposition sequence scheme; the numbers indicate the order of exposition.

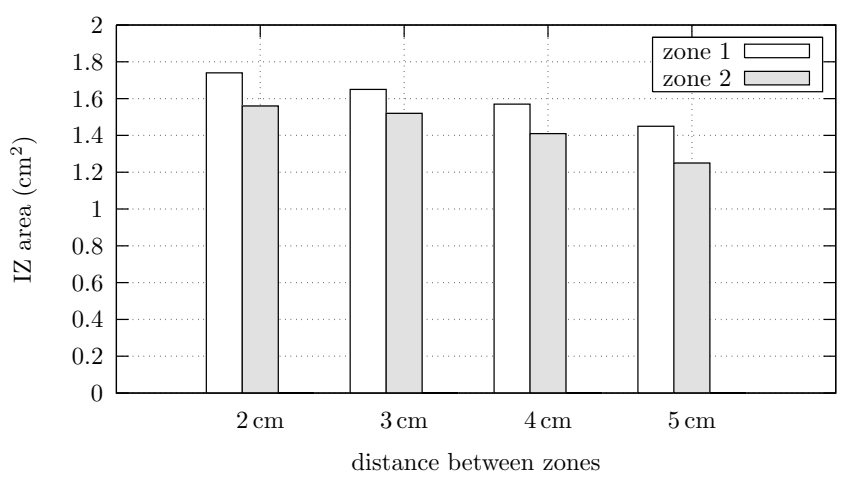

FiguRE 4. The inhibition zone sizes for areas 1 and 2 .

tree times, but the uncertainty of these measurements was smaller than the inhibition zone evaluation uncertainty. That's why the combined uncertainty was given dominantly by uncertainty type B so it is not specified in graphs.

\subsection{TWO EXPOSITIONS INTERACTION}

The differences of both inhibition zone areas were not significant, but the decreasing tendency for the increasing distance between exposed areas was still observed (Fig. 4). The inhibition zone size was determined using computer aided filling of the area by regular grid. Sporadic colonies appeared inside the zones and their edges, indicating the incomplete $C$. albicabs inhibition. The edge of continual growth was considered as the border of particular inhibition zones.

We used distances of $1,2,3,4$ and $5 \mathrm{~cm}$ for the experiment. The overlapping of inhibition zones was observed for the distance of $1 \mathrm{~cm}$, where it was difficult to distinguish particular inhibition zones, therefore this sample was excluded from the data processing.

\subsection{Four EXPOSITIONS WITH SYMMETRIC CLOCKWISE SEQUENCE ORDER}

This is the experiment with three subsequent expositions for distances of $1,2,3,4$ and $5 \mathrm{~cm}$ between opposite exposed sites. The overlapping of inhibition zones was observed for small distances (1 and $2 \mathrm{~cm}$ ), therefore these samples were excluded from data processing (Fig. 5).

The first exposed zone is affected by far treatment of the second and the fourth exposition in this geometric arrangement. The third exposition is farther away, thus effect of this exposition is expected to be smaller. For other expositions applies the similar.

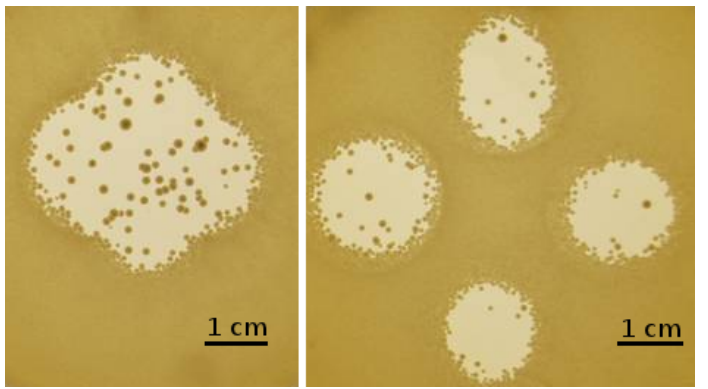

Figure 5. Overlapping of inhibition zones at small distances between exposition sites; left $2 \mathrm{~cm}$, right $4 \mathrm{~cm}$ distance between opposite expositions.

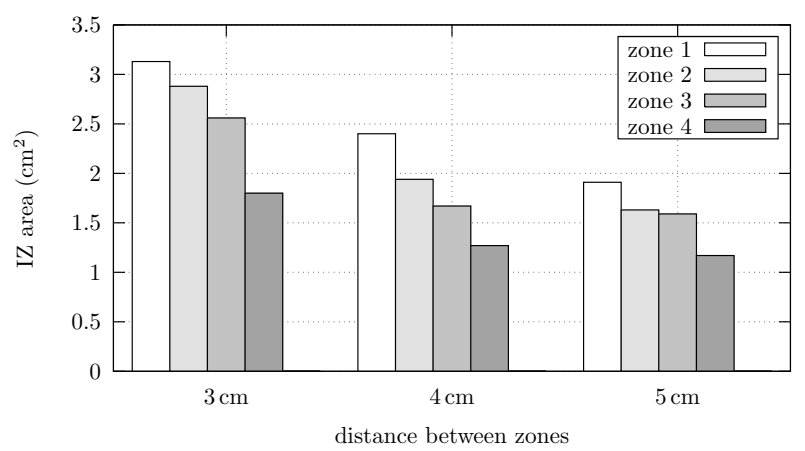

Figure 6 . The inhibition zones sizes for various distances between expositions.

The following mechanisms are present in both experiments: near treatment, far pre-treatment, far posttreatment. We suppose that all of them contribute to overall decontamination with certain rate. It can be seen from both experiments that the first exposed zone was always the biggest one on the Petri dish. This indicates that the far pre-treatment is not as important as the far post-treatment of previously exposed area. The far post-treatment could be mediated by stable particles produced by discharge, ozone $\mathrm{O}_{3}$ seems to be the most probable candidate. The additional effect of UV radiation may be also considerable. Nevertheless, the concentration of ozone was not measured here; concerning its production, see, e.g., 2]. This holds also for UV radiation: we did not dispose any method to measure its intensity.

\section{Conclusions}

The growth of yeast could be inhibited by near and far discharge exposition. The aim of this study was to serially test the gradual increase of Candida albicans growth inhibition zone size caused by increasing discharge exposition sequence. The firstly exposed zone was the biggest one on the Petri dish. It is obvious that the near treatment is the most important. However, impact of the far post-treatment is also significant. The inhibition zone area can be enlarged up by tens percent by far post-treatment.

We can also conclude that the far and the near effects are not commutative. It seems that the far pre-treatment affects yeasts only a little bit. The near 
treatment by high energetic species has naturally the biggest impact for the decontamination. However, the low energetic species, which reach the previously near treated area, seem to be sufficient to finish Candida albicans cells off.

This experiment also showed us, that usage of a single Petri dish for several expositions and subsequent comparison of inhibition areas may cause methodical errors in results.

\section{ACKNOWLEDGEMENTS}

This work was supported by the Czech Technical University in Prague grant SGS13/194/OHK3/3T/13 and Charles University in Prague Research programs PRVOUKP25/LF1/2 and SVV-2012-264506.

\section{REFERENCES}

[1] J. Jorgensen, M. Ferraro. Antimicrobial susceptibility testing: A review of general principles and contemporary practices. Clinical infectious diseases 49(11):1749-55, 2009.

[2] J. Julák, V. Scholtz, S. Kotúčová, O. Janoušková. The persistent microbicidal effect in water exposed to the corona discharge. Physica Medica 28(3):230-239, 2012.

[3] M. Kong, G. Kroesen, G. Morfill, et al. Plasma medicine: An introductory review. New Journal of Physics 11(11):115012, 2009.

[4] Laroussi M. Evaluation of the roles of reactive species, heat, and UV radiation in the inactivation of bacterial cells by air plasmas at atmospheric pressure. International Journal of Mass Spectrometry 233(1-3):81-86, 2004

[5] Z. Machala, L. Chladekova, M. Pelach. Plasma agents in bio-decontamination by DC discharges in atmospheric air. Journal of Physics D: Applied Physics 43(22):222001, 2010.

[6] S. Schneider, J. Lackmann, D. Ellerweg, et al. The role of VUV radiation in the inactivation of bacteria with an atmospheric pressure plasma jet. Plasma Processes and Polymers 9(6):561-568, 2012.

[7] V. Scholtz, J. Julák, V. Kř́iha. The microbicidal effect of low-temperature plasma generated by corona discharge: Comparison of various microorganisms on an agar surface or in aqueous suspension. Plasma Process Polym 7(3-4):237-243, 2010.

[8] V. Scholtz, J. Julák, V. Kř́iha, et al. Decontamination effects of low-temperature plasma generated by corona discharge. Part II: new insights. Prague Med Rep 108(2):128-146, 2007.

[9] T. Shimizu, T. Nosenko, G. E. Morfill, et al. Characterization of low-temperature microwave plasma treatment with and without UV light for disinfection. Plasma Processes and Polymers 7(3-4):288-293, 2010.

[10] E. Stoffels, Y. Sakiyama, D. Graves. Cold atmospheric plasma: Charged species and their interactions with cells and tissues. IEEE Transactions on Plasma Science 36(4):1441-1457, 2008. 